hep-ph/9511350, UW/PT 95-18

\title{
A Viable Model of Dynamical Supersymmetry Breaking in the Hidden Sector
}

\author{
Ann E. Nelson \\ Department of Physics, Box 351560 \\ University of Washington, Seattle, WA 98195-1560
}

\begin{abstract}
We propose a simple and natural model of dynamical supersymmetry breaking, which could be used as a mechanism for spontaneous supersymmetry breaking in a gravitationally coupled hidden sector. The gaugino masses in the visible sector are naturally of the same size as the squark, slepton, and gravitino masses.
\end{abstract}

$11 / 95$ 


\section{Introduction}

Dynamical Supersymmetry Breaking (DSB) [1] is an attractive explanation of the large hierarchy of scales introduced to describe fundamental physics. The usual minimal supersymmetric extension of the standard model, known as the MSSM, is based on the assumption that supersymmetry is spontaneously broken at a scale of $\sim 10^{11} \mathrm{GeV}$ in a "hidden" sector, with soft supersymmetry breaking mass terms of order $\sim 10^{2} \mathrm{GeV}$ arising in the visible sector from supergravitational effects. The MSSM scenario still requires the input of a large $\left(10^{8}\right)$ hierarchy between the Planck and supersymmetry breaking scales, which could be explained by DSB. The aim of this paper is to give an explicit example of supersymmetry breaking in the hidden sector with the following attributes:

1. The supersymmetry breaking in the hidden sector is dynamical. The only explicit scale in the theory is the Planck mass $m_{P}$, with any other scales deriving from dimensional transmutation 1 .

2. The supersymmetry breaking is described by an effective supersymmetric gauge theory with a stable, nonsupersymmetric ground state at field strengths which are much smaller than $m_{P}$.

3. Visible sector gaugino masses of the same size as the squark and slepton masses can arise naturally, which translates into a requirement that the hidden sector should contain a gauge singlet field with an F term as large as any other F terms in the theory.

The reader may be sceptical about the necessity of this exercise as there are already several proposals in the literature for hidden sector dynamical supersymmetry breaking. However the existing models all fail to satisfy either the second or the third criterion 2 . Our rational for imposing these criteria is as follows.

1 Except for a constant in the superpotential needed to tune the cosmological constant to zero. We hope that such tuning might eventually be described by effects within a nonsupersymmetric effective theory of quantum gravity, and will not depend on the details of the effective Lagrangian.

2 While this work was in the process of completion I learned about some overlapping independent work on the subject of hidden sector DSB by R. Plesser and Y. Nir [2]. 
Most proposed hidden sector DSB models have a ground state at field strengths which are as large or larger than the Planck scale. Furthermore it is frequently stated that in these models supersymmetry is spontaneously broken by supergravitational effects. One should be able to redefine the field content of these models to study the effective theory of light excitations about the ground state, which in the reformulated theory occurs for fields with small expectation values and conventional kinetic energy terms. This effective theory should be supersymmetric and should describe spontaneous supersymmetry breaking at a scale well below the Planck scale. Then there is a simple argument, due to Weinberg [3] that in such an effective theory the supersymmetry breaking will persist in the flat space limit where the effects of supergravity are turned off. In the presence of supergravity the scalar potential is $[$ 国

$$
\begin{aligned}
V= & e^{8 \pi K / m_{P}^{2}}\left(\left(K_{i}^{j}\right)^{-1}\left(W_{i}+8 \pi K_{i} W / m_{P}^{2}\right)\left(W_{j}+8 \pi K_{j} W / m_{P}^{2}\right)^{*}-24 \pi|W|^{2} / m_{P}^{2}\right) \\
& +\frac{1}{2} \sum_{A}\left|K_{i} t_{A j}^{i} \phi^{j}\right|^{2} .
\end{aligned}
$$

Here $W$ is the superpotential, $K$ is the Kähler potential, and the $\phi^{i}$ are chiral superfields. If the equations

$$
\begin{aligned}
& \left(W_{i}+8 \pi K_{i} W / m_{P}^{2}\right)=0, \text { all } i \\
& K_{i} t_{A j}^{i} \phi^{j}=0
\end{aligned}
$$

have a solution then this solution is both supersymmetric and an extremum of the potential (1.1), so supersymmetry is not broken. Weinberg argues that if a solution for a globally supersymmetric ground state exists

$$
\begin{aligned}
& W_{i}=0 \\
& K_{i} t_{A j}^{i} \phi^{j}=0
\end{aligned}
$$

then a small perturbation of this solution gives a solution to (1.2) which is locally supersymmetric, and which is a local minimum of the potential (1.1). Thus in order to obtain a model with local supersymmetry breaking one should first find a globally supersymmetric theory without a supersymmetric ground state. Therefore in this letter we focus on breaking global supersymmetry spontaneously. Supergravity can then be treated as a perturbation which leads to small but important effects, such as soft supersymmetry breaking in the visible sector. 
It is well known [5] that global supersymmetry breaking can occur at tree-level in models which introduce the scale of supersymmetry breaking as a parameter in the tree level Lagrangian - and perhaps this is the correct low energy effective field theory description of some nonperturbative effect arising from Planck scale physics.

We find it more plausible that an effective theory of DSB explains the hierarchy between the supersymmetry breaking and Planck scales. Viable examples of DSB have been constructed where visible renormalizable gauge interactions communicate supersymmetry breaking radiatively to squarks, sleptons and gauginos [6]. However Affleck, Dine and Seiberg pointed out [7] that in hidden sector DSB models it appears to be difficult to give the ordinary gauginos a reasonable mass. A gaugino mass term must come from the following term in the effective supersymmetric Lagrangian

$$
\int d^{2} \theta f\left(X_{i} / m_{P}\right) W_{\alpha} W^{\alpha}+\text { h.c. }
$$

where $f$, the gauge kinetic function, is a holomorphic function of $X_{i}$, the chiral superfields. Supergravitational (and other nonrenormalizable effects aring from Planck scale physics) gives squark and slepton masses of order the gravitino mass $m_{3 / 2} \sim\left(1 / m_{P}\right)$. Phenomenologically viable gaugino masses should also arise at $\mathcal{O}\left(1 / m_{P}\right)$ and so $f$ should contain a term linear in a chiral superfield which has an F term of order the supersymmetry breaking scale squared. Such a gauge kinetic function can only be gauge invariant if this superfield is a gauge singlet. However in known DSB models there are no gauge singlets participating in the DSB dynamics, and finding new DSB models, especially ones with gauge singlets, is nontrivial [8].

One possibility [9] is to gauge a global symmetry of a known DSB model 3 and then use this new gauge group to feed supersymmetry breaking radiatively to a gauge singlet. Then this gauge singlet obtains an F-term at one loop, and one expects gaugino masses in the visible sector to be suppressed relative to squark and slepton masses by this loop factor. It would be interesting to know, however, whether such light gaugino masses 4 are

3 Note that simply gauging a global symmetry of a DSB model never restores supersymmetry, as argued in ref. [10].

4 There remains a phenomenologically viable window for such light gaugino masses [1]. Other ways of extending the visible sector to give the gauginos a mass were studied in [12]. 
a necessary consequence of hidden sector DSB.

In the next section we will show how in a modified version of a known DSB model, gauge singlets do get $\mathrm{F}$ terms as large as $\mathcal{O}\left(M_{s}^{2}\right) \sim m_{3 / 2} m_{P}$, and Planck scale physics can easily produce ordinary gaugino masses as large as the squark and slepton masses.

\section{A Hidden Sector Model of Dynamical Supersymmetry Breaking}

Consider the gauge group $\mathrm{SU}(7)$ with matter content

$$
A=21, \quad \bar{F}_{i}=\overline{7}, i=1,2,3,
$$

( $A$ is the two-index antisymmetric tensor).

The model was originally studied by Affleck, Dine and Seiberg (ADS) [7]. They noted that no superpotential could be dynamically generated, and that in the absence of a tree level superpotential there is a classically flat direction with

$$
\begin{aligned}
\bar{F}_{1} & =(1 / \sqrt{3})\left(\begin{array}{lllllll}
\phi & 0 & 0 & 0 & 0 & 0 & 0
\end{array}\right), \\
\bar{F}_{2} & =(1 / \sqrt{3})\left(\begin{array}{lllllll}
0 & \phi & 0 & 0 & 0 & 0 & 0
\end{array}\right), \\
\bar{F}_{3} & =0, \\
A^{\dagger} A & =\operatorname{diag}\left(\left|\phi^{2}\right| / 6,\left|\phi^{2}\right| / 6,0,0,0,0,0\right),
\end{aligned}
$$

(global SU(3) rotations of this direction are also flat). For $\phi$ large, the low energy effective theory includes SU(5) with a $\overline{5}+10$, and a light gauge singlet "dilaton" field $\phi$. The scale at which the $\mathrm{SU}(5)$ theory becomes strong is

$$
\Lambda_{5}=\phi^{-4 / 13} \Lambda_{7}^{17 / 13}
$$

Since there is strong evidence that the SU(5) effective theory dynamically breaks supersymmetry at a scale $M_{s} \sim \Lambda_{5}$ [13] we expect that the dilaton slides off to infinity and the theory has no ground state. ADS then noted that if one adds a superpotential, e.g.

$$
W=A \bar{F}_{2} \bar{F}_{3}
$$

then there are no classically flat directions, and there is a stable vacuum with supersymmetry spontaneously broken. Complete quantitative understanding of the dynamics of the 
supersymmetry breaking and the particle spectrum involves strong interactions and is not "calculable".

Let us now take this $\mathrm{SU}(7)$ theory, (without the superpotential (2.4)), and add 3 gauge singlet fields

$$
X_{i}, i=1,2,3
$$

with (nonrenormalizable) superpotential couplings

$$
W=\left(1 / m_{P}\right) \sum_{i, j, k=1,2,3} \epsilon_{i j k} \lambda_{i} X_{i} A \bar{F}_{j} \bar{F}_{k}
$$

This theory has no classical flat directions involving SU(7)-colored fields although the $X$ 's are undetermined classically when $\mathrm{SU}(7)$ is unbroken. Note that that mass and self coupling terms for the $X$ 's can be eliminated by an $\mathrm{R}$ symmetry under which the $X$ 's have zero charge, and this $\mathrm{R}$ symmetry allows the gauge kinetic functions to depend on the $X_{i}$ 's 5 . A dimension four term such as (2.4) can be made zero by shifting the $X_{i}$ fields.

Now there is competition between two couplings. The $\mathrm{SU}(7)$ dynamics would like $\phi$ to be large in order to reduce the scale of supersymmetry breaking. However due to the superpotential (2.6), assuming the $\lambda_{i}$ 's are all of order one, there is always an $\mathrm{F}$ term for one linear combination of the $X$ 's which has size $\phi^{3} / m_{P}$. The potential is minimized for $\phi$ of order

$$
\phi \sim \Lambda_{7}^{(34 / 47)} m_{P}^{(13 / 47)}
$$

which gives a supersymmetry breaking at a scale

$$
M_{s} \sim \phi^{3} / m_{P} \sim \Lambda_{7}^{(51 / 47)} m_{P}^{(-4 / 47)}
$$

In Appendix A we show that for $\phi$ non zero, the potential also eliminates the flat directions for the scalar components of $X_{i}$. The tree level potential gives the $X$ scalars a mass of order

$$
M_{X} \sim M_{s}^{2} / \phi
$$

The $\mathrm{F}$ term for one linear combination of the gauge singlets $X_{i}$ is of the same order as the supersymmetry breaking scale and provided that the ordinary gauge kinetic function

5 We thank Michael Dine for this comment. 
contains a term like (1.4) which is linear in the $X$ 's the ordinary gauginos will get a mass of the same size as the supergravity-induced squark and slepton masses. If $\Lambda_{7}$ is about $\sim 10^{11} \mathrm{GeV}$, then the gravitino mass will be of order $10^{2} \mathrm{GeV}$. Superpartners in the visible sector will have masses of order the weak scale. The hidden sector scalars $X$ have masses around $3 \times 10^{7} \mathrm{GeV}$ and do not lead to any "Polonyi-type" [9, 14] cosmological problems 6 . There is a Goldstone boson, due to spontaneous $\mathrm{R}$ symmetry breaking at a scale $\phi \sim 10^{13}$ $\mathrm{GeV}$. This Goldstone boson could play the role of an invisible axion [15] in the ordinary QCD sector and solve the strong CP problem. However we expect to softly break the R symmetry by explicitly adding a constant to the superpotential in order to fine-tune the cosmological constant to zero - this explicit symmetry breaking will give the axion a large mass [16], rendering the axion irrelevant both for cosmology and for the strong CP problem.

Now that we have an explicit example, we can present an algorithm for finding models of DSB with gauge singlets. Take a chiral gauge theory which is known to have D flat directions and no supersymmetric ground state, add a number of gauge singlet superfields equal to the number of flat directions, and add a tree level superpotential which couples the gauge singlets to the gauge invariant polynomials of chiral superfields which parametrize the flat directions [17], so that the only remaining classically flat directions have unbroken gauge symmetry. Most of the resulting models do not have a stable supersymmetry breaking ground state, but our example is not the unique DSB model which could serve as a supersymmetry breaking hidden sector.

\section{A Dilaton Problem?}

We have not included the dilaton predicted by string theory, and so do not have the "dilaton problem" of a supersymmetric ground state [18] at infinite dilaton field strength. If we assume our model arises as the low energy limit of a string theory we must also assume that some other mechanism (not associated with supersymmetry breaking) stabilizes the

6 The analysis of hidden sector scalar masses in ref. [9] does not apply here or to any model where the flat directions are lifted by nonrenormalizable terms in the superpotential. 
superstring dilaton]. One might worry 8 that since our $X_{i}$ superfields have dilaton-like couplings to gauge fields, as in eq. (1.4), there is an direction associated with some $X_{i} \rightarrow \infty$ in which supersymmetry tends to be restored. However, unlike in the case of the superstring dilaton, we have no theoretical reason to severely constrain the $X_{i}$ couplings, and so there is no way to decide, (without input from short distance physics) whether the gauge couplings get stronger or weaker as $X_{i} \rightarrow \infty$, and whether supersymmetry is restored in this limit. In any case, the contribution to the mass squared of the $X_{i}$ particles near the origin of field space from the dependence of the gauge coupling constant on the vev $\left\langle X_{i}\right\rangle$ is of order

$$
\left.\left.\Delta M_{X_{i}}^{2} \sim \frac{\partial^{2} M_{s}^{4}}{\left(\partial X_{i}\right)^{2}}\right|_{X_{i}=0} \sim \frac{\partial^{2} \Lambda_{5}^{4}}{\left(\partial X_{i}\right)^{2}}\right|_{X_{i}=0} \sim \frac{M_{s}^{4}}{m_{P}^{2}}
$$

which is smaller by a factor of $\phi^{2} / m_{P}^{2} \sim 10^{-10}$ than the contributions (eq. (2.9)) we have already considered.

\section{Discussion}

We have shown that it is possible to find an effective theory which dynamically breaks supersymmetry in a hidden sector, has a stable ground state with all field expectation values well below the Planck mass, and which can naturally give visible sector gaugino masses of the same order as the squark and slepton masses. The model does not suffer from any of the cosmological problems associated with very light, gravitationally coupled scalars. We see no experimental consequences of this model other than the usual ones associated with supersymmetric extensions of the standard model - in particular we do not see how to compute rather than estimate the superpartner masses, and this construction does not help explain the absence of flavor changing neutral currents 9 . Our model does serve as an existence proof that the assumptions leading to the MSSM can be realized in a self-consistent effective field theory exhibiting dynamical supersymmetry breaking.

7 For instance in some models with two or more gauge groups and no matter, there is a supersymmetric minimum with a stable dilaton, [19]. We could simply add a such a dilaton stabilizing sector to our theory.

8 We thank M. Dine for correspondance on this point.

9 If we assume the validity of string theory and can find an extension of this construction which involves the superstring dilaton in the DSB dynamics, we could gain predictivity [20]. 


\section{Acknowledgements}

I would like to thank Michael Peskin for a conversation which inspired this work, Michael Dine for many useful comments, Joe Polchinski for a discussion about supergravitational effects, and Yosef Nir and Ronen Plesser for sending me a note about their work in

progress. This work was supported in part by the DOE under grant \#DE-FG06-91ER40614 and by the Alfred P. Sloan Foundation.

\section{Appendix A. Potential for Light Scalars}

The classical scalar potential may be derived from the superpotential (2.6). The vaccuum state lies along an SU(7) D flat direction, with some field strengths well above the scale $\Lambda_{7}$, where the effective theory has gauge group $\mathrm{SU}(5)$ and the following chiral superfields: a light $10+\overline{5}, X_{1,2,3}$, and $3 \mathrm{SU}(5)$ gauge singlet chiral superfields. The effective theory breaks supersymmetry dynamically at the $\mathrm{SU}(5)$ scale. If we consider the effective theory at a short distance scale where it is weakly coupled, we can use the classical potential derived from (2.6) to find the dilaton and $X_{i}$ scalar masses. For simplicity we taking $\lambda_{1}=\sqrt{2}=\lambda_{2}=\sqrt{2} \lambda_{3}$, and expand about the D flat directions:

$$
\begin{aligned}
& A=\left(\begin{array}{ccccccc}
0 & \phi / \sqrt{6} & 0 & 0 & 0 & 0 & 0 \\
-\phi / \sqrt{6} & 0 & 0 & 0 & 0 & 0 & 0 \\
0 & 0 & 0 & 0 & 0 & 0 & 0 \\
0 & 0 & 0 & 0 & 0 & 0 & 0 \\
0 & 0 & 0 & 0 & 0 & 0 & 0 \\
0 & 0 & 0 & 0 & 0 & 0 & 0 \\
0 & 0 & 0 & 0 & 0 & 0 & 0
\end{array}\right)
\end{aligned}
$$

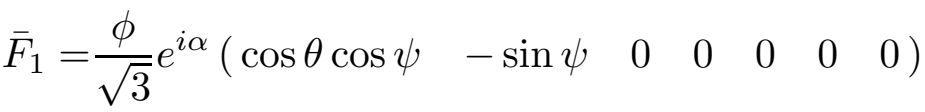

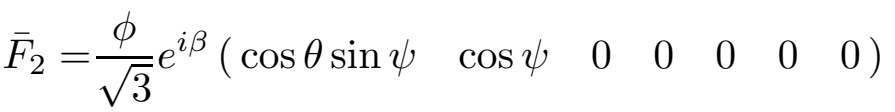

$$
\begin{aligned}
& \bar{F}_{3}=\frac{\phi}{\sqrt{3}}\left(\begin{array}{lllllll}
\sin \theta & 0 & 0 & 0 & 0 & 0 & 0
\end{array}\right),
\end{aligned}
$$


$(\theta, \psi, \alpha$ and $\beta$ are real $)$. We find the potential

$$
\begin{aligned}
V_{\text {classical }}= & \left|F_{X_{1}}\right|^{2}+\left|F_{X_{2}}\right|^{2}+\left|F_{X_{3}}\right|^{2}+\left|F_{A_{12}}\right|^{2} \\
& +\left|F_{\bar{F}_{1}^{1}}\right|^{2}+\left|F_{\bar{F}_{1}^{2}}\right|^{2}+\left|F_{\bar{F}_{2}^{1}}\right|^{2}+\left|F_{\bar{F}_{2}^{2}}\right|^{2}+\left|F_{\bar{F}_{3}^{1}}\right|^{2}+\left|F_{\bar{F}_{3}^{2}}\right|^{2} \\
& \text { (note that all other F terms are zero) } \\
= & \left|\frac{\phi^{6}\left(1-\frac{1}{3} \cos (2 \theta)\right)}{9 m_{P}^{2}}\right| \\
& +\left|\frac{2 \phi^{4}}{9 m_{P}^{2}}\right|\left\{\left|X_{3}\right|^{2}\left(1+2 \cos ^{2} \theta\right)+2\left|X_{1}\right|^{2}\left(1+2 \cos ^{2} \psi \sin ^{2} \theta\right)\right. \\
& +2\left|X_{2}\right|^{2}\left(1+2 \sin ^{2} \psi \sin ^{2} \theta\right)+4 \operatorname{Re}\left(X_{1} X_{2}^{*} e^{i(\alpha-\beta)}\right) \sin (2 \psi) \sin ^{2} \theta \\
& \left.-2 \sqrt{2} \sin (2 \theta) \operatorname{Re}\left[X_{3}^{*}\left(X_{1} e^{i \alpha} \cos \psi+X_{2} e^{i \beta} \sin \psi\right)\right]\right\} .
\end{aligned}
$$

If classical physics were the whole story this potential would just drive the theory to a supersymmetric state with $\phi=0$. However DSB from the SU(5) gauge theory gives a contribution

$$
\Delta V \sim \Lambda_{5}^{4} \sim|\phi|^{-16 / 13} \Lambda_{7}^{68 / 13}
$$

to the potential (note that eq. (A.3) is valid only for $|\phi| \gg \Lambda_{7}$ ), and the potential is minimized for $\phi \sim \Lambda_{7}^{34 / 47} m_{P}^{13 / 47}, \theta=0, X_{1,2,3}=0$, with all noncompact flat directions lifted. Most scalars obtain mass of order $\phi^{2} / m_{P} \sim M_{s}^{2} / \phi$ from the classical superpotential, except those associated with 4 compact flat directions of the potential, which are the result of the spontaneous breakdown of exact global symmetries of the superpotential. Either nonrenormalizable terms in the Kähler potential, or still higher dimension terms in the superpotential, or supergravitational effects, could explicitly break these global symmetries and lead to substantial pseudo Goldstone boson masses. 


\section{References}

[1] E. Witten, Nucl. Phys. B188 (1981) 513

[2] R. Plesser, Y. Nir, private communication

[3] S. Weinberg, Phys. Rev. Lett. 48 (1982) 1776

[4] E. Cremmer, S. Ferrara, L. Girardello, A. Van Proeyen, Phys. Lett. 116B (1982) 231;

R. Barbieri, S. Ferrara, C.A. Savoy, Phys. Lett. 119B (1982) 343

[5] P. Fayet, J. Iliopoulos, Phys. Lett. B51 (1974) 461; L. O’Raifeartaigh, Nucl. Phys. B96 (1975) 331;

[6] M. Dine, A. E. Nelson, Y. Nir, Y. Shirman, to be published in Phys. Rev. D, hep$\mathrm{ph} / 9507378$

[7] I. Affleck, M. Dine, N. Seiberg, Nucl. Phys. B256 (1985) 557

[8] E. Witten, Nucl .Phys. B202 (1982) 253

[9] T. Banks, D. B. Kaplan, A. E. Nelson, Phys. Rev. D49 (1994) 779, hep-ph/9308292

[10] A. E. Nelson, N. Seiberg, Nucl. Phys. B416 (1994) 46, hep-ph/9309299

[11] G. R. Farrar, hep-ph/9508292, hep-ph/9508291; J.L. Feng, N. Polonsky, S. Thomas, hep-ph/9511324

[12] L.J. Hall, L. Randall, Nucl. Phys. B352 (1991) 289; M. Dine, D. MacIntire, Phys. Rev. D46 (1992) 2594, hep-ph/9205227

[13] I. Affleck, M. Dine, N. Seiberg, Phys. Lett. 137B (1984) 187; H. Murayama, Phys. Lett. B355 (1995) 187, hep-th/9505082; E. Poppitz, S. P. Trivedi, preprint EFI-95-44 (Jul 1995) hep-th/9507169

[14] B. de Carlos, J.A. Casas, F. Quevedo, E. Roulet, Phys. Lett. B318 (1993) 447, hepph/9308325; T. Banks, M. Berkooz, P.J. Steinhardt, Phys. Rev. D52 (1995) 705, hepth/9501053; T. Banks, M. Berkooz, S.H. Shenker, G. Moore, P.J. Steinhardt, hepth/9503114

[15] R. Peccei, H. Quinn, Phys. Rev. Lett. 38 (1977) 1440; Phys. Rev. D16 (1977) 1791; S. Weinberg, Phys. Rev. Lett. 40 (1978) 223; F. Wilczek, Phys. Rev. Lett. 46 (1978) 279; J. E. Kim, Phys. Rev. Lett. 43 (1979) 103; M.A. Shifman, A.I. Vainshtein, V.I. Zakharov, Nucl. Phys. B166 (1980) 493; M. Dine, W. Fischler, M. Srednicki, Phys. Lett. 104BB (1981) 199

[16] J. Bagger, E. Poppitz, L. Randall, Nucl. Phys. B426 (1994) 3, hep-ph/9405345

[17] D. Mumford and J. Fogarty, Geometric Invariant Theory (Springer, 1982); M.A. Luty, W. Taylor IV, preprint MIT-CTP-2440 (June, 1995), hep-th/9506098

[18] M. Dine, N. Seiberg, Phys. Lett. 162B (1985) 299

[19] N.V. Krasnikov, Phys. Lett. B193 (1987) 37, T.R. Taylor Phys. Lett. B252 (1990) 59

[20] V. S. Kaplunovsky, J. Louis, Phys. Lett. B306 (1993) 269, hep-th/9303040; J. Louis, Y. Nir, Nucl. Phys. B447 (1995) 18, hep-ph/9411429 